Article

\title{
Preventing, Reducing and Ending LGBTQ2S Youth Homelessness: The Need for Targeted Strategies
}

\author{
Alex Abramovich \\ Institute for Mental Health Policy Research, Centre for Addiction and Mental Health (CAMH), Toronto, ON M5S 2S1, Canada; \\ E-Mail: Alex.Abramovich@camh.ca
}

Submitted: 9 May 2016 | Accepted: 21 September 2016 | Published: 20 October 2016

\begin{abstract}
Gender non-conforming and sexual minority youth are overrepresented in the homeless youth population and are frequently discriminated against in shelters and youth serving organizations. This paper provides a contextual understanding of the ways that institutional and governmental policies and standards often perpetuate the social exclusion of lesbian, gay, bisexual, transgender, queer, and 2-Spirit (LGBTQ2S) youth, by further oppression and marginalization. Factors, including institutional erasure, homophobic and transphobic violence, and discrimination that is rarely dealt with, addressed, or even noticed by shelter workers, make it especially difficult for LGBTQ2S youth experiencing homelessness to access support services, resulting in a situation where they feel safer on the streets than in shelters and housing programs. This paper draws on data from a qualitative Critical Action Research study that investigated the experiences of a group of LGBTQ2S homeless youth and the perspectives of staff in shelters through one-on-one interviews in Toronto, Canada. One of the main recommendations of the study included the need for governmental policy to address LGBTQ2S youth homelessness. A case study is shared to illustrate how the Government of Alberta has put this recommendation into practice by prioritizing LGBTQ2S youth homelessness in their provincial plan to end youth homelessness. The case study draws on informal and formal data, including group activities, questions, and surveys that were collected during a symposium on LGBTQ2S youth homelessness. This paper provides an overview of a current political, social justice, and public health concern, and contributes knowledge to an under researched field of study by highlighting concrete ways to prevent, reduce, and end LGBTQ2S youth homelessness.
\end{abstract}

\section{Keywords}

homelessness; homophobia; LGBTQ2S youth; policy change; social inclusion; transphobia

\section{Issue}

This article is part of the issue "Homelessness and Social Inclusion", edited by Isobel Anderson (University of Stirling, UK), Maša Filipovič Hrast (University of Ljubljana, Slovenia), and Joe Finnerty (University College Cork, Ireland).

(C) 2016 by the author; licensee Cogitatio (Lisbon, Portugal). This article is licensed under a Creative Commons Attribution 4.0 International License (CC BY).

\section{Introduction}

This paper provides a contextual understanding of the ways that institutional and governmental policies and standards often further oppress, marginalize, and perpetuate the social exclusion of lesbian, gay, bisexual, transgender, queer, questioning, and $2-$ Spirit $^{1}$ (LGBTQ2S) youth. The acronym "LGBTQ2S" is used throughout this paper to refer collectively to the wide range of gender and sexual identities that individuals identify with and is meant to represent gender and sexual diversity. Although there may be intersections among the various identity categories, each identity has its own unique needs and experiences. The terms "trans" and "queer" are used interchangeably with LGBTQ2S throughout this paper. The term "trans" (transgender) is used as an umbrella term to describe people who do not conform or identify with the sex assigned to them at birth. Whereas,

\footnotetext{
${ }^{1}$ The term "2-Spirit" refers to Aboriginal people who identify with both a masculine and feminine spirit. This term is not exclusive to gender identity, and can also refer to sexual identity, and spiritual identity (Taylor, 2009).
} 
the term "queer" is a multi-faceted term that has been reclaimed by LGBTQ2S people as an identity category for those who do not identify with binary terms that describe sexual, gender, and political identities (Jagose, 1996). When we deviate from the norm and do not conform to hegemonic identity categories, we are likely to be pathologized and/or labeled (Anzaldúa, 1987; Burstow \& Weitz, 1988). The Diagnostic and Statistical Manual of Mental Disorders (DSM) classified homosexuality as a "mental disorder" up until 1973 (Cooper, 2004). Although homosexuality was removed over 40 years ago, the DSM-5 still pathologizes and labels individuals who have identities that do not fit into the gender binary, with the label "Gender Dysphoria", formerly named "Gender Identity Disorder (GID)" (Moran, 2013). The pathologization of homosexuality and gender non-conformity has led to stereotypes, stigma, homophobia, transphobia, and the exclusion of LGBTQ2S individuals in many spheres of daily living. For example, as Sue (2010) argues, LGBTQ2S individuals are often gawked at in public spaces and are recipients of daily insults and derogatory comments. These intentional and unintentional day-to-day negative attitudes and stigma towards LGBTQ2S individuals can be described as microaggressions (Sue, 2010).

This paper draws on data from a qualitative Critical Action Research study (Carson, 1990) that investigated the experiences of a group of LGBTQ2S homeless youth ( $n=11$ ) between the ages of $16-29$ years old in shelters, and the perspectives and understanding of shelter workers $(n=14)$ and program managers $(n=8)$ in Toronto, Canada, through semi-structured one-on-one interviews and focus groups. Youth participants were initially recruited through recruitment posters in a wide variety of shelters and population-based support services, as an attempt to recruit a diverse group of young people, however, not enough participants were recruited through this method; leading to the administration of snowball sampling and purposive sampling techniques. Maximum variation and opportunistic sampling were administered to ensure that shelter workers and program managers had a diverse range of comfort levels in dealing with situations of homophobia and transphobia in the shelter system, training experiences, and number of years of professional work experience. Critical Action Research combines critical theory and action research in an effort to create practical social change (Given, 2008). A major goal of the study was to share knowledge to help improve the condition of shelters and the policies that rule them, in an effort to provide LGBTQ2S youth with safe, affirming, and accepting services to turn to for support. One of the key recommendations of the study included the need for governmental policy to address LGBTQ2S youth homelessness. This paper shares a case study that illustrates how the Government of Alberta has put this recommendation into practice by prioritizing LGBTQ2S youth homelessness in their provincial plan to end youth homelessness. Youth and staff quotes collected through the Criti- cal Action Research study are shared throughout the paper to exemplify the different ways that the issues presented affect the young people at the center of this work.

Over the past two decades, society's acceptance of sexual and gender diversity has grown, and consequently, youth are coming out at younger ages than ever (Lepischak, 2004; Savin-Williams \& Diamond, 2000). Nonetheless, many young people continue to encounter microaggressions, homophobic and transphobic violence, and discrimination in their personal, familial, and professional lives. One of the most frequently cited pathways leading youth into homelessness is family conflict, regardless of gender or sexual identity (Cull, Platzer, \& Balloch, 2006; Gaetz, 2014; Hagan, \& McCarthy, 1997; Karabanow, 2004). However, identity-based family conflict resulting from a young person coming out as LGBTQ2S is the most prevalent cause of homelessness among queer and trans youth (Abramovich, 2012; Choi, Wilson, Shelton, \& Gates, 2015; Cochran, Stewart, Ginzler, \& Cauce, 2002). Compared to their heterosexual and cisgender ${ }^{2}$ counterparts, LGBTQ2S youth face increased risk of physical and sexual exploitation, mental health difficulties, substance use, HIV risk behaviours, and suicide (Denomme-Welch, Pyne, \& Scanolon, 2008; Durso \& Gates, 2012; Ray, 2006). Further, LGBTQ2S youth commonly experience homophobic and transphobic discriminiation when accessing youth serving organizations, emergency shelters, and housing programs (Abramovich, 2013; Denomme-Welch et al., 2008; Ray, 2006; Tyler, 2013). Factors such as institutional erasure, homophobic and transphobic violence, and discrimination that is rarely dealt with, addressed, or even noticed by shelter workers and management, make it especially difficult for LGBTQ2S youth experiencing homelessness to access shelters, resulting in a situation where they feel safer on the streets than in shelters and support services (Abramovich, 2013; Keurghlian, Shtasel, \& Bassuk, 2014).

Family rejection, inadequate social services, and discrimination in housing, employment, and education, result in situations where LGBTQ2S youth are unable to secure safe and affirming places to live. Transgender youth, especially young transgender women of colour, are amongst the most discriminated against groups of people in housing programs and shelters (Grant et al., 2011; Mottet \& Ohel, 2003). They are often faced with intersecting oppressions, such as transphobia, racism, and homophobia. Due to gaps in knowledge and a lack of reported incidents, discrimination against LGBTQ2S homeless youth is rarely acknowledged or even noticed by shelter workers, shelter management, and policy makers (Abramovich, 2013; Josephson \& Wright, 2000). Research on youth homelessness has continuously cited the overrepresentation of LGBTQ2S young people (Abramovich, 2012; Dunne, Prendergast, \&Telford, 2002; Durso \& Gates, 2012; Ray 2006). One Canadian study, approximatley 16 years ago, estimated that $25-40 \%$ of homeless youth identify as LGBTQ2S

\footnotetext{
2 The term "cisgender" refers to people whose lived gender identity matches with the sex (female/male) they were assigned at birth.
} 
(Josephson \& Wright, 2000). However, large-scale data collection remains limited, which is why provincial and national measurements of LGBTQ2S youth homelessness are often based on older data. The hazard of relying on old data, include that there is an under-estimate of the real prevalence. Without an accurate measurement it is difficult to confirm crucial characteristics of the population, secure necessary increases in funding, or build a policy case for the delivery of more targeted services. This gap in data inevitably impairs service delivery.

A major challenge in accurately measuring LGBTQ2S youth homelessness is that programs and services often do not collect data on gender and sexual identity. Key forms (e.g. intake forms) typically do not include LGBTQ2S identities, particularly transgender and gender non-binary identities. Up until fairly recently, research, government, and community efforts to conduct homelessness counts and street-needs assessments left out important questions regarding LGBTQ2S identity, resulting in minimal understanding of the correlation between coming out and homelessness, the challenges that youth face in trying to form their gender and sexual identities ${ }^{3}$, and the unique needs and challenges experienced by LGBTQ2S youth. Another major challenge in accurately measuring the prevalence of LGBTQ2S youth experiencing homelessness is the issue of hidden homelessness, which refers to individuals who do not access shelters or housing programs, but are living in precarious housing situations, such as couch surfing (Canadian Homelessness Research Network, 2012). Hidden homelessness is a significant concern for this population of young people, especially for those living in rural and remote communities. Targeted youth homelessness strategies that prioritize the diverse challenges and needs of subpopulations of youth that are disproportionately represented amongst homeless youth, including LGBTQ2S youth, are a necessary approach in order to appropriately address youth homelessness.

\section{Institutional Erasure and Invisibility of LGBTQ2S Youth}

There are numerous methods by which LGBTQ2S youth are excluded and made invisible in shelters and housing programs. Institutional and governmental policies and standards frequently further oppress, marginalize, and exclude LGBTQ2S youth. It is critical to understand how institutions and institutional policies, such as shelters and shelter standards, can work to erase LGBTQ2S individuals by not including them in key forms, reports, and the day-to-day operation of programs. Institutional rules and policies that do not consider or include LGBTQ2S identities, particularly transgender identities, play a major role in rendering them invisible and therby erasing them. Namaste (2000) describes institutional erasure as the "conceptual and institutional relations" (p. 137) that render transgender individuals invisible and nonexistent. She argues that the use of "men" and "women" dismisses the possibility that trans people could even exist. Similarily, Serano (2007) argues that by labeling people as either male or female, trans people are erased from public awareness, ignored, and viewed by cisgender individuals as nonentities. Erasure begins when youth first enter the shelter and undergo a formal intake process, where they are asked a series of standard questions. These questions help staff determine if youth belong in the shelter and if they do, then which floor of the shelter they will be placed (male vs. female)-already an erasure. When services do not allow people to self-identify on key forms and only provide the option for people to identify as "male" or "female", any identity that does not fall into the gender binary is not included or documented. One of the youth interviewed, spoke at length about how he attempted to come out as trans during the intake process at a shelter:

"The intake was so shitty in terms of trans stuff. There's just no room for trans or even LGBTQ stuff on their intake. I tried to incorporate it in, 'cause they are like, 'do you need subway tokens to go to your appointments?' and I'm like 'yes! I'm going to this trans program Monday, this trans program Tuesday...' and they just kind of ignored that." (J. J., 26 years old, Critical Action Research Study)

The exclusion of LGBTQ2S identities from key institutional forms and questionnaires creates challenges in accurately measuring the prevalence of LGBTQ2S youth experiencing homelessness, and determining the need for specialized services. Heteronormative ${ }^{4}$ and cisnormative beliefs - such as the assumption that all people born female will identify as women, and that all people born male will identify as men-typically rule gendered spaces, such as the shelter system. Serano (2007) argues that everyday gendering and cisnormative assumptions facilitate the majority of trans-erasure. Shelters are often segregated by male and female sleeping and living corridors, and male and female bathrooms and showers; this enforcement of gender conformity is another form of institutional erasure. The expectation that shelter residents will fit into the gender binary makes the shelter system an especially difficult place for transgender and gender non-conforming individuals. The following quote illustrates how the manager of a youth program described the way that trans-erasure occurs in shelters:

"The fact that there are only men's and women's shelters, the fact that youth shelters have boys and girls'

\footnotetext{
${ }^{3}$ Numerous studies have clumped transgender people under the label sexual minority. While, gender and sexual identity overlap, they are not the same. Gender identity refers to how an individual identifies their gender (male, female, genderqueer, genderfluid, transgender, etc.) and sexual identity refers to how an individual identifies whom they are sexually attracted to (lesbian, gay, bisexual, heterosexual, etc.).

${ }^{4}$ Heteronormative beliefs assume that all people are heterosexual, unless told otherwise, and that heterosexuality is the normal and preferable sexual orientation for everyone.
} 
dorms, and they have boys and girls' bathrooms. Then any time a trans person shows up it's an anomaly, it's like 'whoa, what do we do with you?'. Hopefully that will start to be different if the city keeps track of trans residents. There should be more than one box, they should be keeping track of MTF and FTM, but they're not. That's the type of thing we can say, 'ah ha, there's some kind of need'. Otherwise trans people are completely absent. People who don't exist, don't need access to services, you know, there's no case to be made." (Youth Program Manager, Critical Action Research Study)

As a response to the institutional policies and culture of shelters, and to prevent being stigmatized and excluded, LGBTQ2S youth frequently avoid shelters altogether, or do not feel safe coming out as LGBTQ2S and attempt to hide their identities. For example, one young person stated:

“It was a women's shelter and I didn't feel comfortable there because I didn't identify as female. I stayed one night and then I just stuck with friends." (Kelly, 27 years old, Critical Action Research Study)

The decision to not come out can be understood as a survival strategy, youth also engage in passing as straight and/or cisgender in order to feel safe in the shelter sys- tem and as a way to try to obtain the privilege afforded to those who conform to heteronormative and cisnormative rules. Even though choosing to pass as heterosexual and/or cisgender, still there are implications when youth are forced to do so in order to get support from the very systems that are meant to help all youth. When LGBTQ2S youth avoid shelters, not only do they become invisible, but also staff become even less aware of their existence and needs, and therefore do not recognize their inability to provide support or their need for training (Namaste, 2000). There is a cyclical nature to these relations described that result in institutional erasure and invisibility. Firstly, LGBTQ2S cultural competency training ${ }^{5}$ is often not made mandatory for staff working in shelters or youth serving agencies, resulting in staff and management not feeling prepared to intervene in situations of homophobia and transphobia. Secondly, the lack of staff training perpetuates the dangers towards LGBTQ2S youth in the shelter system because not only do staffs not intervene in situations of homophobia and transphobia, but also youths' needs more generally are not met. As a result, LGBTQ2S youth avoid the shelter system, and staff end up knowing even less about how to meet their needs and how to interact with them (see Figure 1 for illustrations of the cyclical nature of the relations described above).

LGBTQ2S youth have distinct and complex needs, however, they are frequently discriminated against and

\footnotetext{
${ }^{5}$ LGBTQ2S cultural competency training is widely used for staff working in health care and social services to increase skills and knowledge regarding the diverse and complex ways that identities intersect and to improve the delivery of services to LGBTQ2S individuals (Margolies, Joo, \& McDavid, 2016).
}

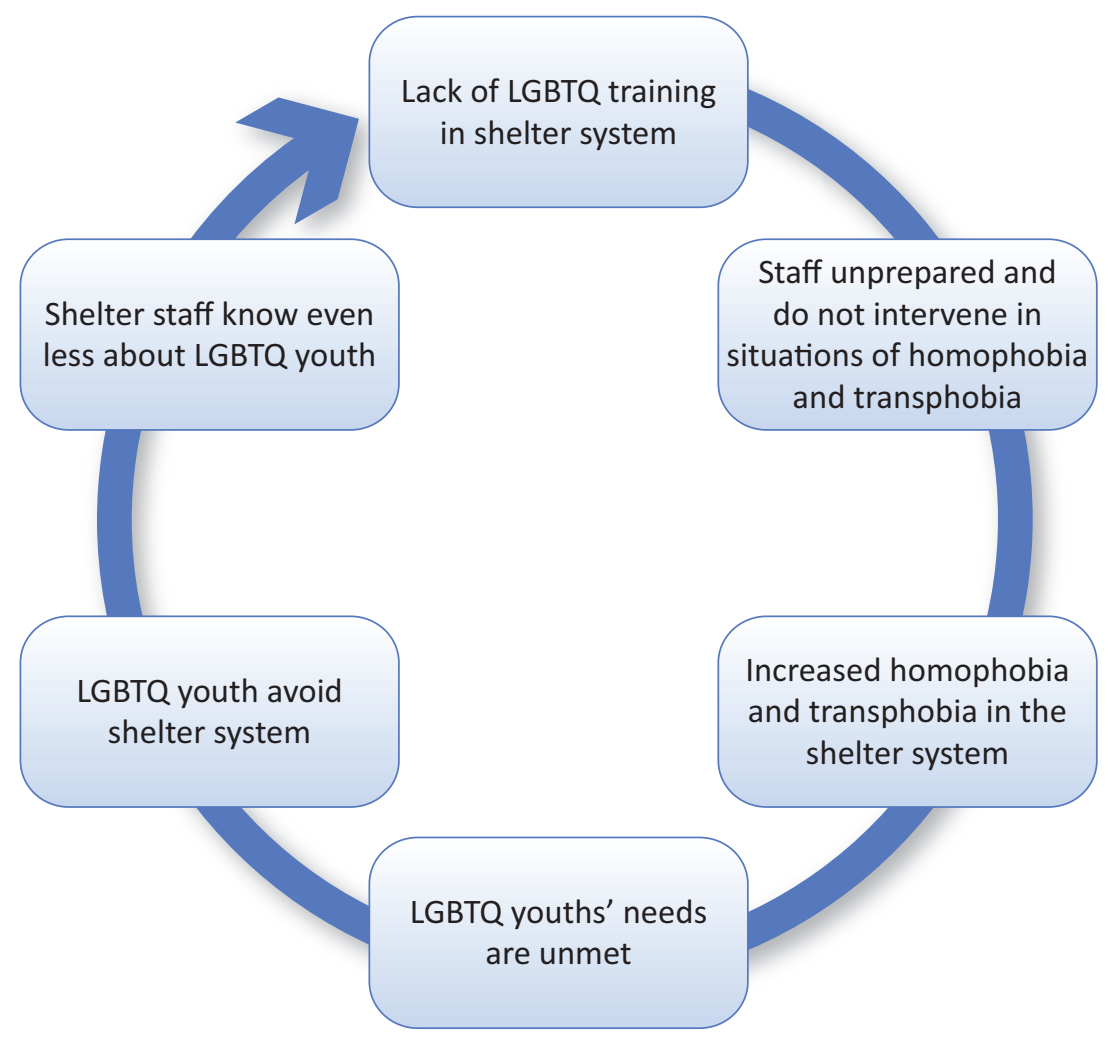

Figure 1. Cyclical nature of the relations. 
excluded from services that are meant to support all young people, regardless of gender and sexual identity. Needs include safe, affirming, and supportive shelter and housing environments, and health care. The needs of transgender youth may differ from those of lesbian, gay, and bisexual youth, for example, transgender youth may choose to start hormones, which requires monitoring, including regular blood work. The lack of specialized health care services for transgender youth often results in youth turning to unmonitored street suppliers for transitionrelated treatment (e.g. hormones, silicone injections), which can have severe health complications (Quintana, Rosenthal, \& Krehely, 2010). The profound impact that homelessness and the lack of support has on LGBTQ2S youth perpetuates issues relating to substance use, risky sexual behaviour, victimization, and crime (Ray, 2006). These issues make LGBTQ2S youth experiencing homelessness even more vulnerable to concerns such as depression and loneliness (Ray, 2006), resulting in a greater need for mental health support. Focused responses and targeted strategies that prioritize LGBTQ2S youth are necessary in order to meet the unique needs of the population and promote social inclusion and acceptance of all identities.

\section{Prioritization of LGBTQ2S Youth}

“You can't just keep creating new systems and new hotlines and fax numbers, you need to go to the root of the problem and create spaces that are queer and trans inclusive.... There needs to be some kind of reporting system or accountability process, some transformative justice happening." (J. J., 26 years old, Critical Action Research Study)

It has been known for over two decades that LGBTQ2S youth are overrepresented amongst the homeless youth population and are frequently unsafe in emergency shelters and housing programs in Canada ( $\mathrm{O}^{\prime}$ Brien, Travers, \& Bell, 1993). However, this issue has only recently entered important dialogue on youth homelessness, both nationally and internationally. It has taken many years to convince key decision makers that LGBTQ2S youth have distinct needs that are often unmet and excluded in shelter services. Support services play a fundamental role in fulfilling homeless youths' daily needs, such as shelter, food, health care, and presumably safety. However, it is essential that services be equipped to deal with the wide-ranging needs of youth, which have undoubtedly become more complex and diverse over the years (Youth Shelter Interagency Network, 2007). Shelters are part of the emergency response to homelessness and are meant to be an entry point for people to gain access to the proper services they need in order to help them out of homelessness and into housing (Raising the Roof, 2009). The inability of shelters to provide safety and support to LGBTQ2S youth is a major barrier in moving this population of youth out of homelessness and into housing.
Support services must be revised and adapted to reflect the changing needs of youth, for example, one youth program co-ordinator stated:

"Systemically there aren't policies that necessarily protect people and talk about inclusion from a useful perspective, address the kinds of barriers that exist for trans people for example. They need policies about access and intake. There need to be policies that say if a trans person comes into the shelter, they will be served in the gender in which they've identified as the safest and most comfortable for them....The onus is on the agency to make the space safer. That needs to be there and that hasn't happened yet." (Youth Program Coordinator, Critical Action Research Study)

Given the range of youth at risk of homelessness or experiencing homelessness, many have diverse, complex, and unique needs that must be recognized and addressed. Which is why preventing, reducing, and ending youth homelessness requires targeted responses for specific subpopulations that are disproportionately represented among homeless and street-involved youth. Prioritizing subpopulations of youth, including LGBTQ2S youth, provides an opportunity to develop targeted responses and strategies that involve critical attention to the unique and diverse needs of the population because the common "one size fits all" approach does not actually work. Even though interest in the issue of LGBTQ2S youth homelessness is growing, still there are minimal specialized housing programs and services that meet the needs of this population in Canada. Focused responses including targeted prevention tactics, specialized housing programs, as well as building the capacity of existing housing programs to serve LGBTQ2S youth in a safe and affirming manner, promote social inclusion and acceptance, and provide a strategy to meet the needs of LGBTQ2S youth. In 2015, the Government of Alberta was the first province in Canada to prioritize LGBTQ2S youth through a targeted plan reflecting the unique needs of LGBTQ2S youth and providing key stakeholders, government, and communities with a common understanding of the causes of homelessness experienced by this population of young people, as well as the needs of LGBTQ2S youth and service providers, and recommendations for solutions.

\section{The Government of Alberta's Youth Plan: A Case Study}

Supporting Healthy and Successful Transitions to Adulthood: A Plan to Prevent and End Youth Homelessness (Youth Plan) was developed to address the unique needs of youth across the province of Alberta, Canada. The Youth Plan has placed particular importance on strengthening and reunifying families whenever possible, ensuring that youth experience healthy transitions across the system of care and within the youth serving sec- 
tor, and preventing youth homelessness. The Youth Plan identified that ending youth homelessness requires the prioritization of subpopulations of young people that are disproportionately represented amongst homeless youth, including LGBTQ2S youth. In order to ensure that LGBTQ2S youth are served more appropriately, the Government of Alberta worked in partnership with a community group and myself (researcher) over the course of ten months, to develop a provincial LGBTQ2S youth strategy that is grounded in evidence-based research, and is rural and urban in focus, given the landscape of communities and services across Alberta. In line with the Youth Plan, emphasis is placed on strengthening families first and ensuring youth experience healthy transitions across the system of care. The prevention of youth homelessness and reunifying families when possible is a top priority.

One of the initial stages of this work involved a provincial youth homelessness symposium, which brought together approximately one hundred service providers, youth workers, managers, and health care professionals to share knowledge and expertise in addressing youth homelessness. Informal and formal data was collected during the symposium, including surveys, group activities, and questions. The data collected provided knowledge regarding the type and frequency of LGBTQ2S cultural competency training offered to shelter workers, housing providers, and youth serving agencies across the province, as well as participants' comfort levels in dealing with situations of homophobia and transphobia in youth serving agencies and shelters, and of course, their perspectives and understanding of LGBTQ2S youth homelessness in Alberta. One of the main outcomes of the provincial youth homelessness symposium was the creation of a provincial LGBTQ2S working group made up of approximately twenty managers of youth serving organizations, government officials, academics, and youth workers (Klingbeil, 2015). Members of the working group were selected by the Alberta Government in order to ensure representation from all parts of the province. The purpose of the working group was to develop policy recommendations and implement program strategies for responding to the needs of LGBTQ2S youth at risk of or experiencing homelessness in the province of Alberta. The work of the working group supported the overarching goals of the Youth Plan through providing strategic policy recommendations on how to respond to the needs of this population of young people. Monthly teleconference meetings were held, encouraging interagency collaboration and the need for community engagement and building partnerships amongst services across the province.

Early work with the working group suggested that rural communities felt isolated in providing support to gender and sexually diverse youth and that there are minimal specialized services available to LGBTQ2S youth in rural communities. Additional findings revealed that LGBTQ2S cultural competency training is not always offered or made mandatory for staff working in youth serving organizations. Unavailability of training makes it dif- ficult for staff to identify homophobia and transphobia, and of course intervene when such incidents occur. Difficulty measuring the prevalence of LGBTQ2S youth accessing services was also revealed, partially due to the absence of standardized policies and procedures, such as provincial or municipal shelter standards, and LGBTQ2S inclusive and affirming intake forms.

An initial report including Short, Medium and Long Term solutions was developed in response to the work of the LGBTQ2S working group and to provide an outline and agenda to launch the prioritization of LGBTQ2S youth homelessness in Alberta. Short-term solutions included recommendations that could be implemented immediately to streamline and prioritize service delivery for LGBTQ2S youth; Medium-term solutions included recommendations requiring system planning and negotiations with funders and agencies to adopt; and Longterm solutions included recommendations requiring policy change or legislation reform to ensure responses and service delivery are tailored to the population. A final report was delivered to the Alberta Government, outlining six key recommendations. Multiple stakeholders were engaged in the development of the recommendations to ensure that the range of LGBTQ2S youth and children receive appropriate supports. Communities and young people need to be involved in the development of strategies and services that are meant to support them, which is why the following recommendations have been greatly driven by community. The implementation of these recommendations will engage government, communities, service providers, educators, parents, and the young people affected most by these issues. The Alberta Government and I worked collaboratively with the working group to develop the following six recommendations, which met the objectives of the Short, Medium and Long-term solutions. The recommendations are meant to help design an effective systemic response to LGBTQ2S youth homelessness and a model of care that is appropriately designed to meet the needs of this population of young people.

\section{Core Recommendations to Alberta Government}

1. Support the delivery of LGBTQ2S specific housing options (development of new housing options and/or refinement of existing housing options):

- Ensuring that there are emergency shelter beds available to LGBTQ2S youth.

- Transitional housing programs.

- Supportive housing programs (Housing First) that are choice focused and place-based (e.g. Host Homes).

2. Support the delivery of population-based programs for LGBTQ2S youth that foster an intersectional approach (development of new programs and/or programs within existing services): 
- Drop-in programs, including: arts, social, cultural, and recreational activities.

- Mentorship programs.

- LGBTQ2S health clinic hours.

- Skill building and employment support.

- Population and/or cultural-specific programming to provide cultural connectedness and access to cultural traditions and practices, including: newcomer/immigrant LGBTQ2S youth; LGBTQ2S youth of colour; and Two-Spirit Indigenous youth.

3. Create provincial housing/shelter standards that focus on working with and meeting the needs of LGBTQ2S young people:

- Standardized gender inclusive intake process (see Figure 2).

- Service providers must respect and accept each client's self-defined gender identity and gender expression, including chosen name and pronoun.

- Gender inclusive washroom policy: Ensuring that all services are equipped with single stall, genderinclusive washrooms (this may be in addition to gendered washrooms in some services) and providing the tools required to convert washrooms, such as signage.

- Guidelines for mandatory and ongoing training, during the first 3 months of hire, for all frontline staff, management, and volunteers of youth serving organizations across the province, as well as foster parents/families fostering LGBTQ2S youth.

- Provincial LGBTQ2S Working Group (continuation of existing working group).

- Supply all shelters, housing programs, and youth serving organizations with appropriate and diverse resources for the young people accessing services, including, pamphlets, fliers, posters on walls, information regarding coming out, LGBTQ2S health, safe sex, as well as information on any local LGBTQ2S services and events. This recommendation requires that staff are made aware of the LGBTQ2S specific programs available, so that they can refer youth to appropriate services when necessary.

- A separate standard regarding access to services for transgender, two-spirit, and gender non-conforming individuals, stating that all shelters/housing programs, and youth serving organizations must accommodate all transgender, two-spirit, and gender non-conforming residents/clients in their selfidentified gender. Services should also be equipped with the appropriate resources and knowledge to refer youth to transition-related treatment (e.g. hormone therapy, legal name change, counseling), and funding and support should be made available for transition related needs.

- A formal grievance/complaints procedure-All shelters/housing programs to implement an internal grievance/complaints process, so that clients/residents can lodge formal anonymous complaints. Clients must be informed of the procedure during the intake process, as well as by posting the grievance/complaints procedure in a conspicuous area of the service.

4. Develop integrated, provincial training solutions for expanded staff training for all aspects of LGBTQ2S cultural competency:

- Expand LGBTQ2S youth homelessness training within all youth serving organizations across the

\author{
How do you describe your sexual identity? \\ Check all that apply:

$\square$ Gay
$\square$ Lesbian
$\square$ Bisexual
$\square$ Queer
$\square$ Pansexual
$\square$ Questioning
$\square$ Straight/Heterosexual
$\square$ Asexual
$\square$ Two-Spirit
$\square$ Identity not listed (please specify)

(n)

\author{
How do you describe your gender identity? \\ Check all that apply: \\ $\square$ Woman \\ $\square$ Man \\ $\square$ Transgender \\ $\square$ Trans woman \\ $\square$ Trans man \\ $\square$ Two-Spirit \\ $\square$ Genderqueer \\ $\square$ Genderfluid \\ $\square$ Androgynous \\ Non-binary \\ $\square$ Cisgender \\ $\square$ Questioning \\ $\square$ Identity not listed (please specify)
}

What gender pronoun do you use?

Figure 2. Standardized intake form questions regarding gender and sexual identity. 
province by: (a) Supporting partnerships between local and/or out of province LGBTQ2S organizations when necessary; (b) Borrowing key principles from best practice guidelines and successful training models.

- Promote the delivery of immediate training to organizations and communities that lack specialized LGBTQ2S resources and have requested support in order to meet the needs of LGBTQ2S youth, such as rural and remote communities.

- LGBTQ2S Cultural Competency Training should include, but not be limited to, the following areas (depending on population served):

- Language/Terminology: Help staff develop more understanding and clarity regarding LGBTQ2S language and terminology, and navigate discussions with comfort and ease.

- Homophobia and Transphobia: Increase understanding and awareness of the causes of homophobia and transphobia and the importance of intervention, as well as the needs, barriers, and experiences of LGBTQ2S homeless youth. Training will help participants identify and intervene when homophobic and transphobic incidents occur, as well as learn how to create safe, secure, and affirming spaces for LGBTQ2S young people.

- Transgender Awareness: Help staff understand how to support young transgender individuals, provide a private space for staff to ask questions regarding working with transgender youth, help organizations create a transgender inclusion policy, and develop trans inclusive and affirming services, become more knowledgeable and develop strategies for reducing barriers for trans service-users.

- Two-Spirit/Indigenous: Increase understanding and awareness of two-spirit identity, and Aboriginal culture and traditions, as well as Aboriginal youth who identify as LGBTQ2S. Training will help service providers create culturally sensitive programs and spaces, and help reduce stigma and discrimination towards LGBTQ2S identified Aboriginal youth.

- Systems Navigation: Ensure that staff members are aware of all local LGBTQ2S resources and programs available for client referrals and education.

5. Develop a prevention plan that emphasizes strategies on early intervention, awareness raising, and programs for children, youth, and families:

- The development of a prevention plan that emphasizes strategies on early intervention, aware- ness raising, and programs for children, youth, and families, and focuses on: (1) Preventing young LGBTQ2S people from becoming homeless; (2) Preventing young LGBTQ2S people from becoming adults experiencing chronic homelessness; (3) Family first/family reconnection (with a supportive family member); (4) Schools with GayStraight Alliances ${ }^{6}$ (GSA) are encouraged to explore the role of GSA's to support LGBTQ2S youth in schools.

- This will involve working collaboratively in a multisystem approach to promote awareness and prepare families, teachers, support workers, heath care professionals, and communities with resources and outreach information, sharing programs so that when young people come out as LGBTQ2S, they are provided with the support they need.

- Placing more emphasis on prevention will help shift the current response to LGBTQ2S youth homelessness from an emergency approach to a longer-term approach, aligning with the Alberta Youth Plan.

6. Develop the capacity for research that frames new approaches and solutions to LGBTQ2S Youth Homelessness:

- Investigation of LGBTQ2S youth homelessness in rural Alberta.

- Evaluation of new and emerging LGBTQ2S programs across the province, which will allow for future LGBTQ2S housing services to operate from an evidence-based model.

- Reassess LGBTQ2S specific questions on measurement procedures and point-in-time counts. Ensure that volunteers conducting counts and surveys receive sensitivity training with regards to asking questions pertaining to gender and sexual identity, and that every respondent is asked about gender identity, and not only the respondents that volunteers perceive as transgender or gender nonconforming. Integrate LGBTQ2S youth with lived experience and LGBTQ2S organizations into the design and execution of counts, which will improve outreach, especially to those who are not accessing services.

- Accurately measuring LGBTQ2S youth homelessness will provide an idea of the prevalence of LGBTQ2S youth homelessness in Alberta and may help us better understand how LGBTQ2S youth move through programs and systems, in order to determine which interventions are working.

- Research that focuses on prevention strategies (e.g. which strategies are successful, which ones should be used, etc.).

\footnotetext{
${ }^{6}$ A Gay-Straight Alliance (GSA) is a student-led club or organization that is intended to provide a safe and supportive space for LGBTQ2S students to support one another and work towards ending homophobia and transphobia in the school system.
} 


\section{Discussion}

The LGBTQ2S youth homelessness strategy emphasizes alignment across government programs and systems, and engages government, communities, and parents and youth, in building solutions. The strategy fosters a standardized model of care for all youth serving agencies, which is necessary in creating accepting, affirming and supportive environments for youth. Enforcing youth serving organizations to conform to the same set of formal rules and regulations will influence service providers to consistently follow standards and create a level of standardization within the youth serving sector, helping youth know what to expect from services and creating a more predictable service system for young people across the province. An integrated provincial LGBTQ2S training plan will help ensure that youth serving organizations are familiar with the needs and experiences of LGBTQ2S youth and help staff navigate discussions with comfort, ease, and understanding. The implementation of inclusive intake forms, close consideration of the physical environment of services (e.g. private and semi-private rooms with washrooms increase access by improving safety), and specialized LGBTQ2S housing options and programs are all critical factors in developing a targeted response to meet the needs of this population of young people and promoting an accepting, affirming, and supportive environment.

LGBTQ2S specialized housing programs are still not recognized as a priority in the majority of governmental policies, however, by supporting the development of LGBTQ2S specific housing options across the province, and developing the capacity for research that frames new approaches and solutions to gender and sexual diversity within the homeless youth population, the Government of Alberta has set a national standard for how to address the issue of LGBTQ2S youth homelessness.

\section{Conclusion}

Proposals and strategies for ending youth homelessness should be comprehensive in scope and need to encompass all of the elements that youth need, not only to survive, but also to thrive. The unique and diverse needs of all young people must be considered and included. We must also find ways to collaborate with the young people who are affected most by these issues because their voices matter and they need to be heard and included in the design of programs and strategies. While the emergency response to youth homelessness remains necessary and important, especially for LGTBQ2S youth who have recently been kicked out of the house after coming out or forced to leave home due to unsafe conditions; there is also a need for strategies that focus on longer-term solutions and on helping young people find and keep housing, as outlined in the recommendations. Traditional prevention strategies typically focus on strategies that keep youth from becoming homeless in the first place. However, it may not always be possible to prevent LGBTQ2S youth from becoming homeless, which is why we need to place more emphasis on helping youth exit the streets, so that they do not become adults experiencing chronic homelessness. Although reuniting LGBTQ2S youth with their parents may not always be an option, there tends to be at least one supportive family member and we need to focus on reuniting youth with those family members. By developing targeted responses that focus on subpopulations of young people that are disproportionately represented among homeless youth, we can change the way that we approach youth homelessness, and ensure that no young person is excluded on the basis of their gender or sexual identity. A comprehensive approach that involves various initiatives is necessary in developing a prevention strategy for this population of youth, as well as continuing to collect data on LGBTQ2S identity and pathways into homelessness. If we learn more about the primary causes and risks, we can better address prevention strategies and work towards ending LGBTQ2S youth homelessness.

This paper has provided an overview of a current political, social justice, and public health concern, and contributes knowledge to an under researched field of study by highlighting concrete ways to prevent, reduce, and end LGBTQ2S youth homelessness.

\section{Acknowledgements}

A very special thanks to the courageous young people who participated in this research-thank you for sharing your important voices. Thank you to my colleague Corey Flanders-Foster, for your insightful comments on an earlier draft.

\section{Conflict of Interests}

The author declares no conflict of interests.

\section{References}

Abramovich, I. A. (2012). No safe place to go: LGBTQ youth homelessness in Canada-Reviewing the literature. Canadian Journal of Family and Youth, 4(1), 29-51.

Abramovich, I. A. (2013). No fixed address: Young, queer, and restless. In. S. Gaetz, B. O'Grady, K. Buccieri, J. Karabanow, \& A. Marsolais (Eds.), Youth homelessness in Canada: Implications for policy and practice. Toronto: Canadian Homelessness Research Network Press. Retrieved from http://www.homelesshub.ca/ resource/23-no-fixed-address-young-queer-and-rest less

Anzaldúa, G. (1987). Borderlands la frontera. San Francisco: Aunt Lute Books.

Burstow, B., \& Weitz, D. (1988). Shrink resistant: The struggle against psychiatry in Canada. Vancouver: New Star Books. 
Canadian Observatory on Homelessness. (2012). Canadian definition of homelessness. Toronto: Homeless Hub. Retrieved from http://www.homelesshub.ca/ homelessdefinition

Carson, T. (1990). What kind of knowing is critical action research? Theory into Practice, XXIX(3), 167-173.

Choi, S. K., Wilson, B. D. M., Shelton, J., \& Gates, G. (2015). Serving our youth 2015: The needs and experiences of lesbian, gay, bisexual, transgender, and questioning youth experiencing homelessness. Los Angeles: The Williams Institute with True Colors Fund.

Cochran, B. N., Stewart, A. J., Ginzler, J. A., \& Cauce, A. M. (2002). Challenges faced by homeless sexual minorities: Comparison of gay, lesbian, bisexual, and transgender homeless adolescents with their heterosexual counterparts. American Journal of Public Health, 92(5), 773-775.

Cooper, R. (2004). What is wrong with the DSM? History of Psychiatry, 15(1), 2-25.

Cull, M., Platzer, H., \& Balloch, S. (2006). Out on my own: Understanding the experiences and needs of homeless lesbian, gay, bisexual and transgender youth. Brighton and Hove: Health and Social Policy Research Centre. Retrieved from http://www.spectrumIgbt.org/downloads/reports/Out_On_My_Own full_report.pdf

Denomme-Welch, S., Pyne, J., \& Scanlon, K. (2008). Invisible men: FTMs and homelessness in Toronto. Toronto: Wellesley Institute. Retrieved from http:// wellesleyinstitute.com/files/invisible-men.pdf

Dunne, G. A., Prendergast, S., \& Telford, D. (2002). Young, gay, homeless and invisible: A growing population? Culture, Health \& Sexuality, 4(1), 103-115.

Durso, L. E., \& Gates, G. J. (2012). Serving our youth: Finding from a National survey of service providers working with lesbian, gay, bisexual, and transgender youth who are homeless or at risk of becoming homeless. Los Angeles: The Williams Institute with True Colors Fund and The Palette Fund.

Gaetz, S. (2014). Coming of age: Reimagining the response to youth homelessness in Canada. Toronto: The Canadian Homelessness Research Network Press.

Given, L. M. (2008). The SAGE encyclopedia of qualitative research methods: Volume 1. Washington, DC: Sage Publications.

Grant, J. M., Mottet, L. A., Tanis, J., Harrison, J., Herman, J. L., \& Keisling, M. (2011). Injustice at every turn: A report of the national transgender discrimination survey. Washington: National Center for Transgender Equality and National Gay and Lesbian Task Force.

Hagan, J., \& McCarthy, B. (1997). Mean streets: Youth crime and homelessness. Cambridge: Cambridge University Press.

Jagose, A. (1996). Queer theory: An introduction. Melbourne: Melbourne University Press.

Josephson, G., \& Wright, A. (2000). Ottawa GLBT wellness project: Literature review and survey instru- ments. Toronto: The Homeless Hub. Retrieved from http://www.homelesshub.ca/Library/Literature-Revi ew-and-Survey-Instruments-54233.aspx

Karabanow, J. (2004). Being young and homeless: Understanding how youth enter and exit street life. New York: Peter Lang.

Keurghlian, A. S., Shtasel, D., \& Bassuk, E. L. (2014). Out on the street: A public health and policy agenda for lesbian, gay, bisexual, and transgender youth who are homeless. American Journal of Orthopsychiatry, 84(1), 66-72.

Klingbeil, A. (2015, January 25). Province creates working group to tackle LGBTQ youth homelessness. Calgary Herald. Retrieved from http://www. calgaryherald.com

Lepischak, B. (2004). Building community for Toronto's lesbian, gay, bisexual, transsexual, and transgender youth. Journal of Gay and Lesbian Services, 16(3/4), 81-98.

Margolies, L., Joo, R., \& McDavid, J. (2016). Best practices in creating and delivering LGBTQ cultural competency trainings for health and social service agencies. New York: National LGBT Cancer Network.

Moran, M. (2013, April 5). New gender dysphoria criteria replace GID. Psychiatric News. Retrieved from http://psychnews.psychiatryonline.org

Mottet, L., \& Ohle, J. (2003). Transitioning our shelters: A guide to making homeless shelters safe for transgender people. New York: The National Coalition for the Homeless and the National Gay and Lesbian Task Force Policy Institute.

Namaste, V. K. (2000). Invisible lives: The erasure of transsexual and transgendered people. Chicago: The University of Chicago Press.

O’Brien, C. A., Travers, R., \& Bell, L. (1993). No safe bed: Lesbian, gay and bisexual youth in residential services. Toronto: Central Toronto Youth Services.

Quintana, N. S., Rosenthal, J., \& Krehely, J. (2010). On the streets: The federal response to gay and transgender homeless youth. Washington: Centre for American Progress. Retrieved from http://www.american progress.org/issues/2010/06/pdf//gbtyouthhomeless ness.pdf

Ray, N. (2006). Lesbian, gay, bisexual and transgender youth: An epidemic of homelessness. Retrieved from http://www.thetaskforce.org

Savin-Williams, R. C., \& Diamond, L. M. (2000). Sexual identity trajectories among sexual-minority youths: Gender comparisons. Sexual Behaviour, 29(6), 607627.

Serano, J. (2007). Whipping girl: A transsexual woman on sexism and the scapegoating of femininity. Berkeley, CA: Seal Press.

Sue, D. W. (2010). Microaggressions, marginality, and oppression: An introduction. In D. Sue (Ed.), Microaggressions and marginality: Manifestation, dynamics, and impact (pp. 3-25). Hoboken, NJ: Wiley.

Taylor, C. (2009). Health and safety issues for Abo- 
riginal transgender/two-spirit people in Manitoba. Canadian Journal of Aboriginal Community-based HIV/AIDS Research, 2, 63-84.

Tyler, K. A. (2013). Homeless youths' HIV risk behaviors with strangers: Investigating the importance of social networks. Archives of Sexual Behavior, 42(8), 15831591.

Yonge Street Mission. (2009). Changing patterns for street involved youth. Toronto: The Yonge Street Mission. Retrieved from http://www.ysm.ca

\section{About the Author}

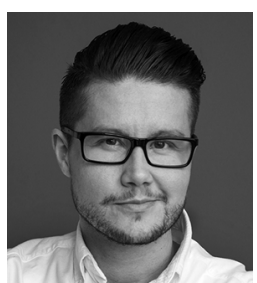

Alex Abramovich (PhD) is an Independent Scientist at the Centre for Addiction and Mental Health (CAMH). Alex has been addressing the issue of LGBTQ2S youth homelessness for over 10 years. He is an internationally recognized leader in the area of LGBTQ2S youth homelessness. Alex's research focuses on the experiences of LGBTQ2S youth in shelters and housing programs; access to mental health services; and how broader policy issues serve to create oppressive contexts for LGBTQ2S youth. 